\title{
Accidental suppression of Landau damping of the transverse breathing mode in elongated Bose-Einstein condensates
}

\author{
B. Jackson and E. Zaremba \\ Department of Physics, Queen's University, Kingston, Ontario KYL 3N6, Canada.
}

(May 15, 2019)

We study transverse radial oscillations of an elongated Bose-Einstein condensate using finite temperature simulations, in the context of a recent experiment at ENS. We demonstrate the existence of a mode corresponding to an inphase collective oscillation of both the condensate and thermal cloud. Excitation of this mode accounts for the very small damping rate observed experimentally, and we find excellent quantitative agreement between experiment and theory. In contrast to other condensate modes, interatomic collisions are found to be the dominant damping mechanism in this case.

PACS numbers: 03.75.Fi, 05.30.Jp, 67.40.Db

The study of collective excitations provides us with a powerful tool for probing the fundamental properties of quantum many-body systems. For example, measurements of low-lying excitations in a Bose-Einstein condensed (BEC) gas of trapped atoms [1] helped establish the Gross-Pitaevskii equation (GPE) as an excellent description of condensate dynamics at low temperatures [2]. At higher temperatures the situation is complicated by the existence of a significant noncondensed fraction. Interactions with this thermal cloud leads to damping and frequency shifts of condensate collective modes, as measured in several experiments [3 6 ]. A theoretical understanding of these processes is important as a foundation for continuing studies in this area, as well as providing a fascinating opportunity for studying quantum systems at finite temperatures.

One can think of interatomic scattering processes as being comprised of mean fields and collisions, where their relative importance in describing the condensate dynamics normally depends upon the rate of collisions compared to the characteristic timescale of the oscillation. When collisions are relatively frequent, the coupled dynamics of the condensate and thermal cloud are most appropriately described by hydrodynamic Landau-Khalatnikov two-fluid equations [7]. It is well known that in liquid helium this leads to hydrodynamic first and second sound modes. In the opposite, collisionless regime, mean field interactions become more important. With the possible exception of Ref. [4], experiments have so far resided in this regime. Here, coupling between fluctuations in the condensate and normal thermal cloud densities leads to Landau damping and an associated frequency shift of the condensate mode, and has been the subject of several theoretical treatments [8,9]. However, the thermal cloud can play a role beyond that of simply providing a damping mechanism. It can undergo collective motion of its own, so that the response of the system to an external probe can give rise to a coupled oscillation of both the condensate and thermal cloud. This kind of mean field coupling is responsible 10,11 for some of the behavior observed in the experiment of Ref. [3].

In this paper we study the transverse breathing mode of a cigar-shaped condensate at finite temperatures, as recently observed in an experiment at ENS [6]. As we shall see, the coupled dynamics of both the condensate and thermal cloud are particularly important in this situation, giving rise to somewhat surprising behavior. Such considerations are crucial in providing an understanding of the very small damping rates and frequency shifts measured in this experiment.

Consider a Bose gas with atoms of mass $m$ confined in an axisymmetic harmonic trap potential of the form $V(\mathbf{r})=m\left[\omega_{\perp}^{2}\left(x^{2}+y^{2}\right)+\omega_{z}^{2} z^{2}\right] / 2$. The ratio between the axial and radial trap frequencies, $\lambda=\omega_{z} / \omega_{\perp}$, characterizes the trap anisotropy. In this work we will apply the formalism of [12], where the dynamics of the system are described by a generalized GPE for the condensate wavefunction, $\Phi(\mathbf{r}, t)$,

$$
i \hbar \frac{\partial \Phi}{\partial t}=\left(-\frac{\hbar^{2}}{2 m} \nabla^{2}+V+g\left[n_{c}+2 \tilde{n}\right]-i R\right) \Phi,
$$

and, within a semiclassical Hartree-Fock approximation, a Boltzmann equation for the thermal cloud phase-space distribution, $f(\mathbf{p}, \mathbf{r}, t)$,

$$
\frac{\partial f}{\partial t}+\frac{\mathbf{p}}{m} \cdot \nabla f-\nabla U_{\mathrm{eff}} \cdot \nabla_{\mathbf{p}} f=C_{12}[f]+C_{22}[f] .
$$

The condensate and thermal cloud densities are given by $n_{c}(\mathbf{r}, t)=|\Phi(\mathbf{r}, t)|^{2}$ and $\tilde{n}(\mathbf{r}, t)=\int\left(\mathrm{d} \mathbf{p} / h^{3}\right) f(\mathbf{p}, \mathbf{r}, t)$, respectively. In the above equations, an important quantity is the interaction parameter $g=4 \pi \hbar^{2} a / m$ (where $a$ is the $s$-wave scattering length). It not only enters into the effective mean field potential $U_{\text {eff }}=V+2 g\left(n_{c}+\tilde{n}\right)$, but also into the collision integrals $C_{22}$ (which represents binary collisions involving only thermal atoms) and $C_{12}$ (involving a condensate atom in the incoming or outgoing channels). The effect of atom exchange in the $C_{12}$ collision process appears in (11) through the non-Hermitian source term $R(\mathbf{r}, t)=\left(\hbar / 2 n_{c}\right) \int\left(\mathrm{d} \mathbf{p} / h^{3}\right) C_{12}[f]$.

First of all, it is instructive to summarize the behavior of the system in the $T=0$ limit and for temperatures above the transition, $T>T_{c}$. The former corresponds to a pure condensate, described by (11) with $\tilde{n}=0$ and $R=$ 
0 . For an axisymmetric anisotropic trap $(\lambda \neq 1)$, analysis of the GPE in the interaction-dominated Thomas-Fermi (TF) limit gives two low-energy $m=0$ modes with frequencies $\omega_{ \pm}^{2} / \omega_{\perp}^{2}=2+3 \lambda^{2} / 2 \pm\left(9 \lambda^{4}-16 \lambda^{2}+16\right)^{1 / 2} / 2$ [2]. The high-lying mode $\omega_{+}$will be of interest here, which for a cigar-shaped trap with $\lambda \ll 1$ corresponds to a breathing-type oscillation in the radial direction, with a frequency $\omega_{+} \rightarrow 2 \omega_{\perp}$ as $\lambda \rightarrow 0$. In this limit the radial oscillations are decoupled from those in the axial direction. Interestingly, for a non-interacting condensate the mode frequency is also $2 \omega_{\perp}$ for all $\lambda$, so that the transverse mode frequency in the $\lambda \rightarrow 0$ limit is independent of interactions. This behavior is similar to that found theoretically for a gas in a 2D harmonic trap interacting with a contact potential [13], where an underlying hidden symmetry gives rise to an undamped breathing mode with frequency $\omega=2 \omega_{\perp}$, independent of interaction strength, temperature, or statistics. For the ENS experiment, $\lambda \simeq 6.46 \times 10^{-2}$, and the high-lying mode has the TF frequency $\omega \simeq 2.00052 \omega_{\perp}$, in good agreement with the experimentally measured value.

Above $T_{c}$, the thermal cloud evolves according to (2) with $n_{c}=0$ and $C_{12}=0$. The mode frequencies can then be calculated by taking moments of the kinetic equation [14]. In the hydrodynamic limit the transverse mode frequency is $\omega_{\mathrm{HD}}=\sqrt{10 / 3} \omega_{\perp}$ 14 15 for $\lambda \rightarrow 0$, while the collisionless result is $\omega_{\mathrm{CL}}=2 \omega_{\perp}$. The ENS experiment resides in the near-collisionless regime, where the mode has frequency $\omega \simeq \omega_{\mathrm{CL}}$ and is weakly damped through interatomic collisions. So, one has the interesting situation where both the condensate at $T=0$ and the thermal cloud at $T>T_{c}$ have mode frequencies close to $2 \omega_{\perp}$.

The question then arises as to the behavior for $0<$ $T<T_{c}$, where both the condensate and thermal cloud are present. Answering this question requires a selfconsistent solution of the coupled GPE (11) and Boltzmann equations (2). We perform this task numerically using a split-operator/Monte-Carlo simulation, as described in 16]. As we shall show, a mode exists at finite temperatures corresponding to an in-phase motion of the two components with a frequency of $\omega \simeq 2 \omega_{\perp}$, which is a direct consequence of the behavior in the $T=0$ and $T>T_{c}$ limits. This mode, which is only weakly damped, corresponds to a breathing oscillation of the entire trapped gas. We demonstrate that this mode was predominately excited in the ENS experiment [6], leading to the anomalously small measured damping rate and frequency shift.

We first discuss results of our simulations for a temperature of $T=125 \mathrm{nK}$, where all other parameters are chosen to match the experiment [6,17. At this temperature, which should be compared to the experimentally measured critical temperature of $T_{c} \simeq 290 \mathrm{nK}$, we find a condensate fraction of 0.56 . We calculate the radial moments, $\left\langle x^{2}+y^{2}\right\rangle=\int \mathrm{d} \mathbf{r} n\left(x^{2}+y^{2}\right)$, as a function of time for both the condensate and thermal clouds. This moment projects out the transverse breathing mode, and is related to the condensate squared radius measured in Ref. [6].

Three distinct excitation schemes are considered. The first matches that employed experimentally, where the radial trap frequency was abruptly changed, then reset to its original value after a time $\tau$. This procedure can be represented by $\omega_{\perp}^{\prime}(t)=\omega_{\perp}\{1+\alpha[\Theta(t)-\Theta(t-\tau)]\}$, where $\Theta(x)$ is the Heaviside step function. In Fig. 1(a) we show results for $\alpha=0.26$ and $\omega_{\perp} \tau=0.172$. The figure clearly shows that both components respond by oscillating with approximately equal amplitudes and phase, at a frequency very close to $2 \omega_{\perp}$. The in-phase oscillation of the condensate and thermal cloud continues over the entire timescale of the simulation, with both amplitudes decaying at a very similar rate. Importantly, this damping is very weak, much smaller than one would expect for Landau damping which is usually the dominant damping mechanism in this regime.

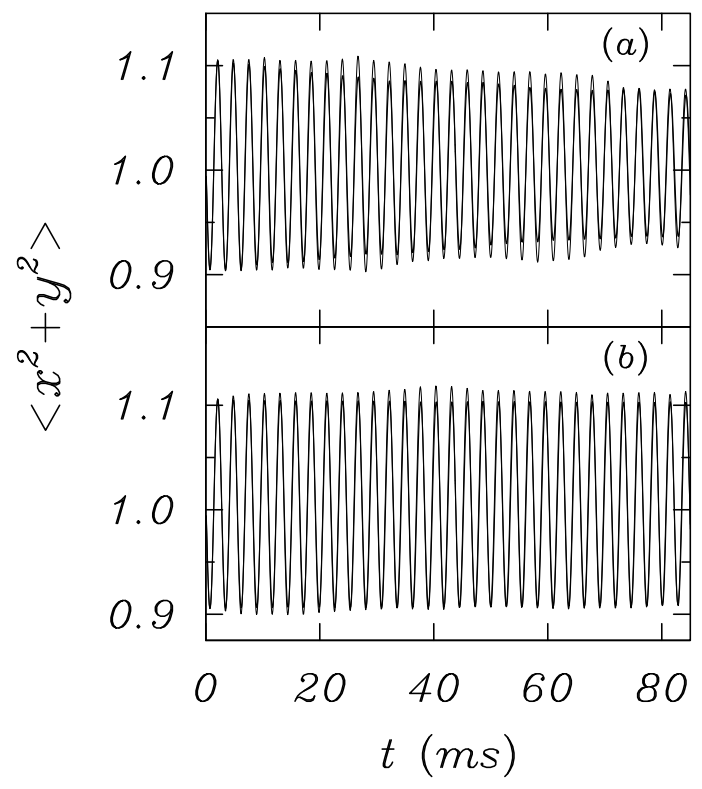

FIG. 1. Time-dependent moment $\left\langle x^{2}+y^{2}\right\rangle$ for the condensate (narrow line) and thermal cloud (bold line), divided by the corresponding values at $t=0$. Data is for a temperature of $T=125 \mathrm{nK}$ and is the result of exciting the system using the tophat perturbation employed experimentally 6]. Simulations shown in (a) include collisions, while those in (b) exclude all collisions $\left(C_{12}=C_{22}=0\right)$.

To understand this behavior one should note that calculations of Landau damping [8,9] conventionally assume that the noncondensate is always in thermal equilibrium. Fluctuations of the condensate mean field induce a response in the thermal cloud density, where the dissipative and reactive components in turn give rise to damping and frequency shifts of the condensate mode. However, this picture is clearly inadequate when the thermal cloud is itself in oscillation. In this case, mean field coupling may lead to the thermal cloud driving the condensate at its own resonant frequency, which can be significant when 
the natural frequencies of the two components are nearly degenerate. As shown in [11], a similar near-resonant driving of the condensate is in fact responsible for the abrupt change in mode frequency observed at high temperatures in Ref. 3]. The present case can be seen as an extreme example of this behavior, as the natural frequencies of the condensate and thermal cloud are both very close to $2 \omega_{\perp}$. Mean field interactions then lead to phase-locking of the oscillations of the two components.

We test this interpretation by separately exciting the two components in our simulations. This is readily achieved by imposing velocity fields at $t=0$ of the form $\mathbf{v} \propto x \hat{\mathbf{i}}+y \hat{\mathbf{j}} 11,18$ on either the condensate or the thermal cloud. Fig. 2(a) shows the result of exciting the condensate only. The condensate oscillation is initially damped quite strongly, while the oscillation in the thermal cloud slowly builds up in time. Eventually, the condensate and thermal cloud settle down to a small amplitude in-phase mode. The initial condensate relaxation is essentially Landau damping due to mean field interaction with the stationary thermal cloud, with a damping rate similar to that found for the $m=2$ mode discussed later. This excitation scheme could be implemented experimentally by optically imprinting a phase onto the condensate, and would be useful in verifying the physical picture presented here.

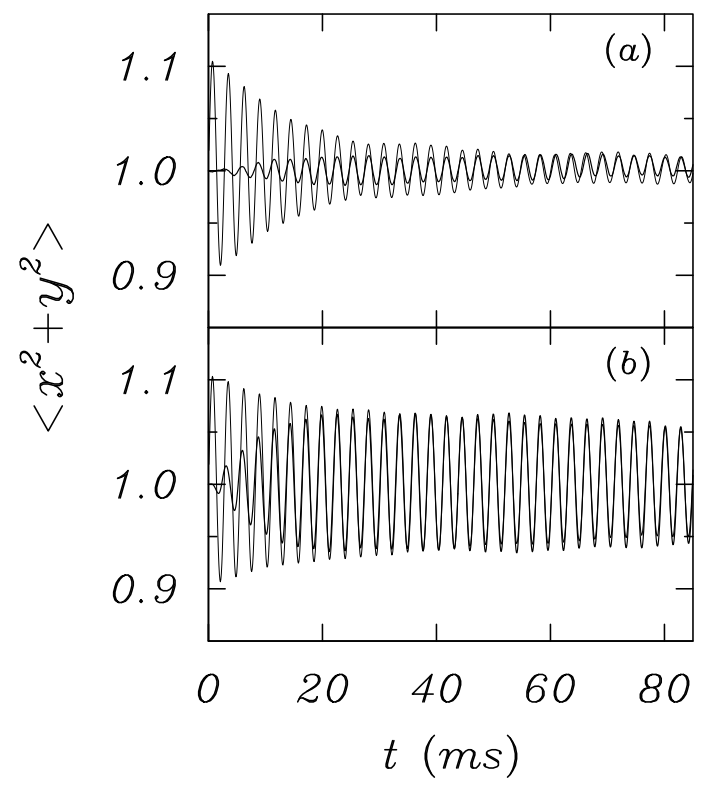

FIG. 2. Results of initially exciting only the (a) condensate, and (b) thermal cloud by imposing velocity fields on each, for a temperature $T=125 \mathrm{nK}$. In (a) the condensate moment is represented by the narrow line with the thermal cloud in bold, while in (b) the line styles are interchanged for clarity.

In Fig. 2(b) we show the result of exciting the thermal cloud only. Again, we see the relaxation of one component (thermal cloud) matched by excitation of the other (condensate), with both settling into an in-phase oscilla- tion at later times. The larger amplitude of the in-phase mode in this case is a consequence of the much greater spatial extent of the thermal cloud. On imposing the same velocity field, the energy increase is proportional to the equilibrium value of $\left\langle x^{2}+y^{2}\right\rangle$, which is several times larger for the thermal cloud than for the condensate at the temperature considered. During the subsequent evolution, the initial energy of either component is redistributed until the two components oscillate together in phase. Since the energy of this in-phase mode is mostly stored in the thermal cloud, one can see that the final amplitude should indeed be larger when the thermal cloud is excited initially.

To compare our results directly to experiment, we fit the condensate data for our first excitation scheme [as displayed in Fig. 1(a)] to an exponentially decaying sinusoid $A \cos (\omega t+\varphi) \mathrm{e}^{-\Gamma t}+C$. Our results for the frequency $\omega$, and damping rate $\Gamma$ 19 are plotted in Fig. 3 for a range of temperatures, together with the corresponding experimental data. We find very good agreement between experiment and theory, for both the frequency and damping rate 20]. It is interesting to note that, along with the very small damping, the frequency is virtually independent of temperature in both simulations and experiment. This is in marked contrast to experimental [3 5] and theoretical results [8,9,11] for other modes, and is a unique feature of this breathing mode excitation. We note that fits to the thermal cloud data give results very similar to those in Fig. 3.

So far we have not addressed the origins of the damping. To provide insight into this question, we have performed a simulation at $T=125 \mathrm{nK}$ where collisions are artificially switched off. Thus, the only coupling between the two components is provided by the mean field terms. The result is plotted in Fig. 1(b). The most striking feature of this plot, when compared to Fig. 1(a), is the almost complete absence of damping, either in the condensate or the thermal cloud. Specifically, a fit to the condensate moment yields a damping rate an order of magnitude smaller than the collisional result. Furthermore, the separate inclusion of either $C_{12}$ or $C_{22}$ collisions yields values intermediate between the collisionless and full collisional results. Finally, artificially increasing the collision cross-section by a factor of two leads to an increase in the damping rate by a corresponding factor. These simulations, together with the excellent agreement found with experiment, provide strong evidence that collisions play a crucial role in determining the observed damping.

We should emphasize that the supression of Landau damping discussed here is specific to the $m=0$ transverse breathing mode in a highly prolate harmonic trap, and is a consequence of the accidental degeneracy between the condensate and thermal cloud frequencies. This condition is no longer satisfied, for example, by the lowest $m=2$ mode, since the condensate now has a frequency of approximately $\sqrt{2} \omega_{\perp}$ [2] while the thermal cloud frequency remains close to $2 \omega_{\perp}$ [14. A simulation 
at $T=125 \mathrm{nK}$ for this mode indeed shows a damping rate of $\Gamma \simeq 50 \mathrm{~s}^{-1}$, an order of magnitude larger than that of the $m=0$ mode and in agreement with the qualitative result mentioned in [6]. Moreover, we would also expect larger damping rates for the $m=0$ mode as $\lambda \rightarrow 1$, since the condensate TF frequency approaches $\sqrt{5} \omega_{\perp}$ in this limit. Simulations at $\lambda=0.57$ and $\lambda=0.75$ confirm that lifting the degeneracy between the condensate and thermal cloud modes leads to an increase in damping with decreasing trap anisotropy.

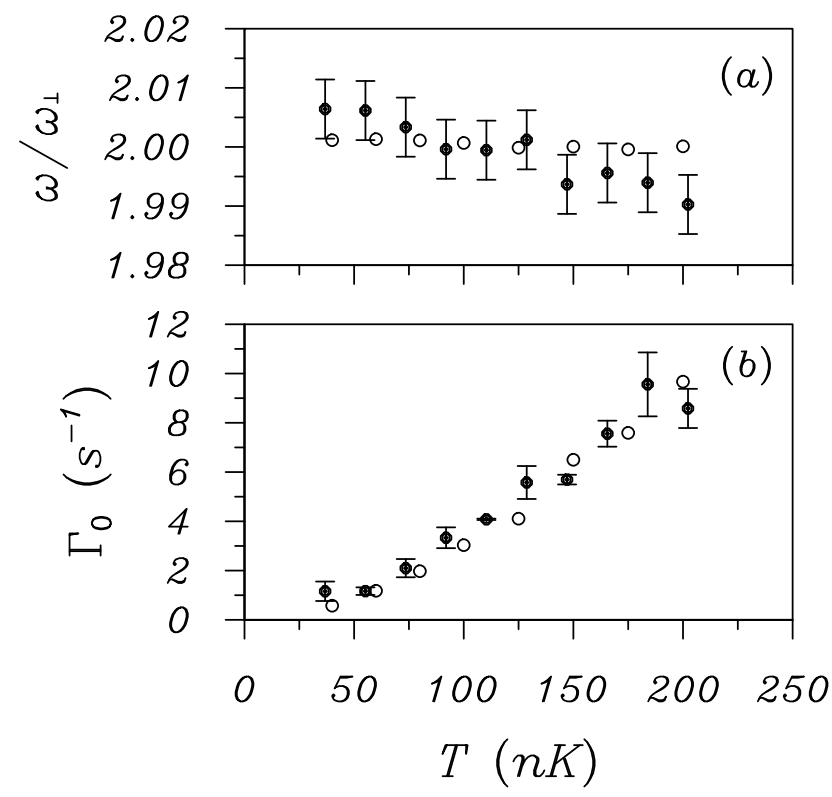

FIG. 3. Frequency (a) and damping rate (b) of the condensate breathing mode. Our results (open circles) are compared to the experimental data (solid circles), where the simulation parameters and excitation scheme are chosen to reproduce the experimental conditions [6].

In summary, we have used finite-temperature simulations to study the transverse breathing mode in elongated Bose-Einstein condensates, as realized in a recent experiment [6]. We show that the very small damping rate and frequency shift observed experimentally is a result of a degeneracy between the condensate and thermal cloud oscillation frequencies, which results in a mode comprised of an in-phase oscillation of both components. The weak damping arises through interatomic binary collisions, rather than mean field interactions (Landau damping) as is normally the case. A comparison of simulation results to experiment shows excellent agreement, confirming the validity of our theoretical approach and underlining the importance of treating the full noncondensate dynamics, including collisions, when accounting for experimental behavior.

We thank V. Bretin for useful information and for providing us with the experimental data in Fig. 3. We acknowledge use of the HPCVL computing facility at Queen's University, and financial support from Natural
Sciences and Engineering Research Council of Canada.

[1] D. S. Jin et al., Phys. Rev. Lett. 77, 420 (1996); M.-O. Mewes et al., ibid. 77, 988 (1996).

[2] S. Stringari, Phys. Rev. Lett., 77, 2360 (1996).

[3] D. S. Jin, M. R. Matthews, J. R. Ensher, C. E. Wieman, and E. A. Cornell, Phys. Rev. Lett. 78, 764 (1997).

[4] D. M. Stamper-Kurn, H.-J. Miesner, S. Inouye, M. R. Andrews, and W. Ketterle, Phys. Rev. Lett. 81, 500 (1998).

[5] O. Maragò, G. Hechenblaikner, E. Hodby, and C. J. Foot, Phys. Rev. Lett. 86, 3938 (2001).

[6] F. Chevy, V. Bretin, P. Rosenbusch, K. W. Madison, and J. Dalibard, Phys. Rev. Lett. 88, 250402 (2002).

[7] T. Nikuni and A. Griffin, Phys. Rev. A 63, 033608 (2001).

[8] W.V. Liu, Phys. Rev. Lett. 79, 4056 (1997); L. P. Pitaevskii and S. Stringari, Phys. Lett. A 235, 398 (1997); P. O. Fedichev, G. V. Shlyapnikov, and J. T. M. Walraven, Phys. Rev. Lett. 80, 2269 (1998); J. Reidl et al., Phys. Rev. A 61, 043606 (2000).

[9] S. Giorgini, Phys. Rev. A 61, 063615 (2000); S. A. Morgan, J. Phys. B 33, 3847 (2000); M. Guilleumas and L. P. Pitaevskii, Phys. Rev. A 61, 013602 (2000); M. Rusch et al., Phys. Rev. Lett. 85, 4844 (2000).

[10] M. J. Bijlsma and H. T. C. Stoof, Phys. Rev. A 60, 3973 (1999).

[11] B. Jackson and E. Zaremba, Phys. Rev. Lett. 88, 180402 (2002).

[12] E. Zaremba, T. Nikuni, and A. Griffin, J. Low Temp. Phys. 116, 277 (1999).

[13] Yu. Kagan, E. L. Surkov, and G. V. Shlyapnikov, Phys. Rev. A 54, R1753 (1996); L. P. Pitaevskii and A. Rosch, ibid. 55, R853 (1997).

[14] D. Guéry-Odelin, F. Zambelli, J. Dalibard, and S. Stringari, Phys. Rev. A 60, 4851 (1999).

[15] A. Griffin, W.-C. Wu, and S. Stringari, Phys. Rev. Lett. 78, 1838 (1997).

[16] B. Jackson and E. Zaremba, preprint cond-mat/0205421.

[17] In particular, we consider ${ }^{87} \mathrm{Rb}$ atoms confined with a radial trap frequency of $\omega_{\perp}=2 \pi \times 182.6 \mathrm{~Hz}$. Experimental use of evaporative cooling meant that the total number of atoms decreased with temperature. We account for this variation in our simulations.

[18] Note that in the limit $\omega_{\perp} \tau \ll 1$ the tophat excitation employed experimentally corresponds to a delta function impulsive perturbation. In linear response this is equivalent to imposing identical velocity fields of the form given on both the condensate and thermal cloud.

[19] Due to small departures of the condensate data from an exponentially-decaying form, our determination of the damping rate has some uncertainty, which we estimate to be about $15 \%$.

[20] Experimentally a condensate amplitude revival was observed at low temperatures and after strong excitations. We see no evidence of this behavior in simulations that 
extend over periods twice as long as those in Fig. 1. Possible sources for this effect are discussed in [6]. 